weakened. The rotation wings accompanying the Rayleigh lines, which show no intensity maximum separated from the centre (Fig. 2,c) in the liquid, develop a distinct maximum at the expected position (Fig. $2, a$ and $b$ ) in the vapour. The absolute intensity of the rotation wing, as judged by comparing it with the 992 Raman line, increases enormously as we pass into the vapour state. For example, the wing accompanying the 4077 line has not been recorded at all in the liquid whereas it is quite intense in the gas (see Fig. 1), in spite of the fact that the 992 Raman line is recorded with greater intensity in the former than in the latter.

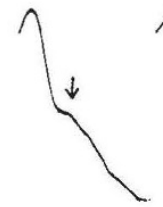

a

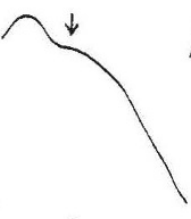

6

Fig. 2 .

The significance of these and other features of the investigation will be discussed by us in detail elsewhere.

S. Bhagavantam.

A. Veerabhadra Rao.

Department of Physics,

Andhra University,

Waltair.

Nov. 16.

'C. K. Ingold and others, J. Chem. Soc., 912-987 (July 1936).

\section{Active Nitrogen}

Is their recent paper on the afterglow in active nitrogen, Cario and Stille ${ }^{1}$ have doubted the reality of the production in the nitrogen afterglow of the $\mathrm{N}_{2}+$ first-negative bands and those bands of the $\mathrm{N}_{2}$ firstpositive system which originate on vibrational levels higher than $v^{\prime}=14$. It is suggested that my results were due to an accidental superposition of light from the exciting discharge on that of the afterglow.

An examination of the afterglow photographs published by Cario and Stille shows immediately the reasons for their failure to observe the above mentioned bands in the afterglow. Their nitrogen was not purified enough for the tube to reach what I have called the auroral stage. Their photographs show the bands due to nitric oxide and cyanogen in the spectrum of the afterglow, whereas these bands, as an examination of my published spectra will show, should have completely disappeared before the auroral stage is reached.

During the preparation of more than a score of tubes, I have passed through the stage photographed by Cario and Stille. There are, at the present time, two tubes in my laboratory which show the stage they have photographed, and several much purer tixbes. In several of the latter type of tubes the negative bands are so strong that they can be observed visually on a direct vision spectroscope. In none of those tubes can we observe the nitric oxide or yanogen bands. These have disappeared because if the purification of the nitrogen.

\section{University of California}

JOSEPH KaPLAN. at Los Angeles.

${ }^{1}$ Cario and Stille, Z. Phys., 102, 317 (1936).

\section{Reflection from the Ionosphere}

THE conditions for the reflection of radio waves from the ionosphere are generally obtained by putting $\mu^{2} \doteqdot 0$. But it is more correct, as was pointed out by Appleton", to obtain this condition by finding out when the group velocity vanishes. If we work out the conditions for the vanishing of the group velocity, neglecting friction, we find certain unexpected results. While for the ordinary wave there is only one condition of reflection given by

$$
p=p_{0} \quad . \quad \text {. . . . }
$$

(where $p$ is pulsatance and $p_{0}^{2}=4 \pi N e^{2} / m$ ), the conditions for the extraordinary wave are found to be as follow :

$$
\begin{aligned}
& p^{2}-p p_{\boldsymbol{H}}=p_{0}{ }^{2} \quad \cdot \quad \cdot \quad(2 a) . \\
& p^{2}+p p_{H}=p_{0}{ }^{2} \quad \text {. } \quad \text {. } \quad(2 b), \\
& p^{2}-p r^{2}=p_{0}^{2} \text { (app.) . (2c), }
\end{aligned}
$$

where $p_{H}=e H / m c, p_{T}=e H_{T} / m c$, when we are using short waves. The first and second of these are those usually obtained for the extraordinary wave reflection. It is usually supposed that (2a) is the only one obtained, as $(2 b)$ is supposed to penetrate and to be absorbed. Toshniwal ${ }^{2}$ was the first to observe and interpret the reflection corresponding to $(2 b)$. This has also been recently confirmed by Leiv Harang ${ }^{3}$ working at Tromsø. The third is a new one and has not been so far reported.

It is well known that for the reflection correspond. ing to $(2 a)$, the difference between the penetration frequencies for the ordinary and the extraordinary waves is approximately $0.7 \mathrm{mc} / \mathrm{sec}$. While this has been confirmed in this laboratory, Messrs. Pant and Bajpai, working after midnight, have several times observed the penetration frequency difference to be only about $0.14 \mathrm{mc} . / \mathrm{sec}$. This result at first appeared to be very puzzling, as it could not be accounted for on the existing theories. But it is easily explained as a reflection given by $(2 c)$. At Allahabad, $p T^{2}=40.5 \times 10^{12}$, and in the neighbourhood of 75 metres the difference between the penetration frequencies is $0.13 \mathrm{mc} . / \mathrm{sec}$. according to $(2 \mathrm{c})$. For higher latitudes (for example, England) this difference will be very small (for 4 megacycles, $0.03 \mathrm{mc} . / \mathrm{sec}$.) and it will be difficult to observe. It would be interesting to know whether similar results are obtained at other tropical stations.

Details of the calculation are being published in the proceedings of the National Institute of Sciences, India.

Physical Laboratory,

$$
\text { R. N. RAI. }
$$

University, Allahabad.

$$
\text { Dec. } 21 .
$$

${ }^{1}$ Appleton, NatoRe, 122, 879 (1928).

Toshniwal, Nature, 135, 437 (1935).

${ }^{3}$ Leiv Harang, Terr. Mag., June 1936.

\section{Who were the Picts?}

Is the note in Nature of December 19 (p. 1061) upon Dr. Bachmakoff's discussion about the evidence of a pre-Aryan population of southern Russia, he is quoted as suggesting that in France the Veneti were a pre-Aryan people "possibly related to the Picts". This lands one in the vexed question whether there ever was a race or tribe that assumed and bore the name Pict. 